\title{
Propuesta de un sistema de radiodifusión digital de alta definición para banda UHF a través de una topología celular
}

\author{
P.R. Ojeda-Cisneros, S. Landeros-Ayala y R. Neri-Vela \\ Red Corporativa de Datos Telmex, División de Estudios de Posgrado y Departamento de \\ Telecomunicaciones Facultad de Ingeniería, UNAM \\ E-mails:pojeda@telmex.com,sland@fi-b.unam.mx,aldeca@fi-b.unam.mx
}

(recibido: octubre de 2002; aceptado: junio de 2003)

\begin{abstract}
Resumen
México se ha caracterizado por ser un país tradicionalmente consumidor (y no generador) de tecnología y estándares. Tratando de modificar esta visión de país, en este trabajo se elaboró una propuesta para la realización de un sistema de transmisión que pueda brindar la televisión del futuro con la mejor calidad posible, considerando su viabilidad de construcción al tomar en consideración el estado de la tecnología actual y contrarrestando especialmente las limitantes propias del canal de transmisión asignado para el servicio de HDTV en México.
\end{abstract}

Descriptores: radiodifusión, alta definición, interferencia, modulación, codificación, ecualización, turbo códigos, multicanalización, distribución, televisión.

\begin{abstract}
Traditionally, Mexico has been known as a consumer (and not a generator) of technology and standards. With the objective of changing this vision of country, this investigation propose a transmission system that can support the future television with the best quality available, considering the viability of the construction using actual technology and counteracting the special limitations of the transmission channel allocated for the HDTV service in Mexico.
\end{abstract}

Keywords: broadcasting, HDTV, interference, OFDM, ATSC, DVB-T, coding, equalization, modulation, multiplexing, CDMA; turbo codes, trellis, television.

\section{Introducción}

Al paso del tiempo se ha intentado mejorar la calidad de la imagen de televisión definiendo nuevos estándares para su transmisión. Contrario a lo que pudiera pensarse, existen varios puntos de con- vergencia en el diseño de estos sistemas; por ejemplo, los propuestos actualmente basan la codificación de video en predicción entre tramas con compensación de movimiento y utilizan la transformada discreta coseno para reducir la correlación entre píxeles vecinos. No sólo esto, sino también han sido 
diseñados para producir imágenes de alta calidad, con el doble de resolución horizontal y vertical que la de los sistemas convencionales, con tasas de transmisión cercanas a los $20 \mathrm{Mb} / \mathrm{s}$. Sin embargo, no sucede así con el diseño de la codificación de canal y la forma de distribuir la señal. Esta diferencia se debe a que muchas de las técnicas de transmisión digital tienen (relativamente) poco tiempo de utilizarse en aplicaciones de radiodifusión y a que los canales que actualmente se ocupan para radiodifusión analógica, presentan diversas limitantes a los sistemas digitales, las cuales se mostrarán a continuación.

\section{Limitantes para los sistemas HDTV}

Los sistemas HDTV se enfrentan a varias limitantes, entre las que se encuentran las siguientes:

Asignación de espectro de radio-frecuencia. El espectro de radiofrecuencia debe ser compartido entre una gran cantidad de aplicaciones, que varían desde uso militar hasta uso doméstico. Diversos organismos de regulación han asignado un segmento del espectro a radiodifusión HDTV terrestre. En México se encuentra en las bandas VHF y UHF, y a su vez, se divide en canales con un ancho de banda de $6 \mathrm{MHz}$.

La interferencia a un servicio existente NTSC, debida a una señal HDTV no debe ser mayor quela interferencia actual entre dos señales NTSC

Ruido. En cualquier enlace por ondas electromagnéticas, como es el caso de la radiodifusión HDTV, se presenta la existencia de ruido.
Multitrayectorias. Es un fenómeno de propagación que ocurre por la circulación de la señal en múltiples rutas, lo que provoca que cada una de ellas llegue a diferente tiempo. Esto sucede por la reflexión de la señal en edificios o montañas cercanas. Esto es común en las bandas de VHF y UHF, también aparece en los llamados "fantasmas" que son imágenes múltiples. Sin embargo, en los sistemas digitales a este fenómeno se le conoce con el nombre de Dispersión temporal y provoca interferencia que produce atenuación, como una función de la frecuencia, a través del ancho de banda de la señal. Para reducir su efecto es necesaria la ecualización.

\section{Fuentes de interferencia}

Las fuentes de interferencia para un receptor sintonizado en un canal de UHF pueden ser diversas. Entre ellas están las siguientes:

Interferencia cocanal. Es provocada por estaciones vecinas que transmiten en la misma frecuencia y tienen otra región de cobertura.

Interferencia por canal adyacente. Sucede por efectos no lineales en el televisor o debido a transmisores cercanos de alta potencia que operen en una banda adyacente de frecuencia.

- Imágenes producidas por las portadoras de sonido y video, o por la frecuencia intermedia.

Intermodulación entre portadoras de distintas estaciones.

Estas fuentes de interferencia se pueden producir de manera lineal (señales de 
interferencia de imágenes) o de forma no lineal (intermodulación). Finalmente, el resultado es el mismo: la imagen (o el sonido) no seve (o se escucha) de la forma en que debiera.

\section{Sistemas de transmisión HDTV}

Los sistemas de transmisión HDTV, deben ser capaces de entregar grandes flujos de información de manera rápida y confiable, aún bajo condiciones precarias del canal. Existen ciertas características comunes a todos los sistemas de transmisión HDTV propuestos. La manera en la que se conforman las características de los sistemas de transmisión se muestra en la figura 1.

Gran parte de la complejidad en el diseño de un sistema de transmisión reside en el receptor. Además de la demodulación y corrección de errores, el receptor debe ser capaz de generar señales de referencia sincronizadas, tanto en fase como en frecuencia, a la portadora utilizada en el proceso de modulación en el transmisor, así como extraer símbolos de temporización para que la señal demodulada pueda ser muestreada en sincronía con el reloj transmisor. En muchos casos, el canal introduce distorsiones provocadas por dispersión y desvanecimiento. Existen varias formas de compensar estos efectos y pueden ser implementadas en el transmisor o en el re- ceptor. Una de estas formas es la ecualización, la cual se implementa en el extremo receptor (Nicolas, 1994).

\section{Propuesta del sistema}

A continuación se mostrarán uno a uno los subsistemas de comunicaciones elegidos para obtener un sistema que utilice de la mejor forma posible el espectro asignado en banda UHF para televisión terrestre, contrarrestando los efectos negativos del medio. El criterio con el que fueron elegidos fue, en la mayoría de los casos, el de la mejor tecnología existente, excepto cuando esto implique una complejidad excesiva que no justifique los beneficios exhibidos. Para finalizar, se mostrará de forma gráfica la estructura de los sistemas de comunicación utilizados en los extremos transmisor y receptor.

\section{Esquema de modulación}

Se ha decidido utilizar un esquema híbrido que conste de una constelación digital de tasa múltiple 64, 16, y 4-PSK no distribuida uniformemente con modulación analógica radial superpuesta, como se muestra en la figura 2. Con este esquema es posible obtener resoluciones aún mayores que las brindadas por los sistemas de HDTV actuales, de hasta $1600 \times 900$ píxeles, cuando

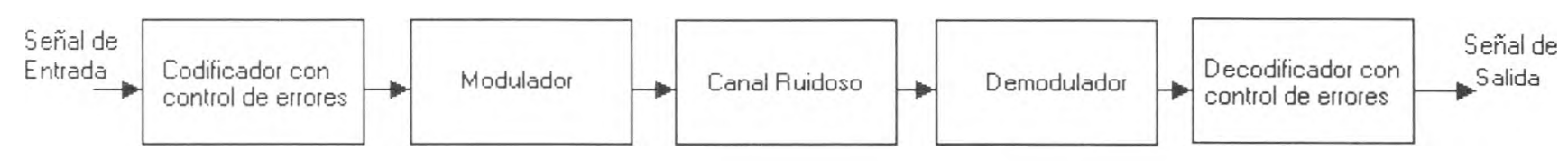

Figura 1. Diagrama de bloques de un sistema de transmisión HDTV 


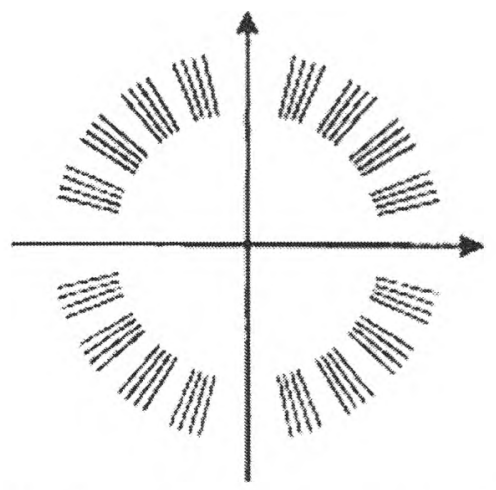

Figura 2. Esquema elegido para el sistema propuesto

se utiliza la totalidad de portadoras disponibles en el espectro (Ojeda, 2001). De esta forma se podrían construir receptores móviles, que generalmente constan de pantallas pequeñas y demoduladores que sólo obtienen señales de nivel 1, con lo que se podrían lograr señales con resoluciones de hasta 1080x607 píxeles a 60 cuadros por segundo con resolución completa, lo que daría una calidad mucho mejor a la televisión digital transmitida actualmente por sistemas de televisión directa vía satélite. Esto no vendría en decremento de la calidad óptima de la imagen, pues en realidad en pantallas menores a 15 pulgadas no se notan diferencias considerables al utilizar resoluciones mayores. La tabla 1 nos describe las características del tipo de modulación propuesta para la creación del sistema.

\section{Codificación de la señal}

Al constar elesquema de modulación con dos componentes, una digitaly otra analógica, es necesario proteger a ambas contra los efectos de la interferencia y el ruido.

Para conformar la componente analógica se propone el uso de un esquema de espectro ensanchado por secuencia directa. De esta forma, cada componente digital verá a la parte que le corresponda del espectro como un ruido en amplitud y la interpretará como tal, sin provocarle problema alguno, ya quela fase y la amplitud son cantidades ortogonales. Además, debido a las propiedades de las técnicas de espectro ensanchado, su distribución de potencia se comportará de forma casi plana con niveles de potencia inferiores a los del nivel de ruido (Figura 3 ).

Para proteger la componente digital, se utiliza un esquema de control y corrección de errores FEC. En este tipo de esquemas se envía redundancia de la señal, de tal forma que ésta permita identificar y corregir errores en el aparato receptor. Con esto se envía una sola señal para todos los receptores, pero cada uno de ellos la utiliza a su conveniencia y de manera distinta, dependiendo de sus necesidades.

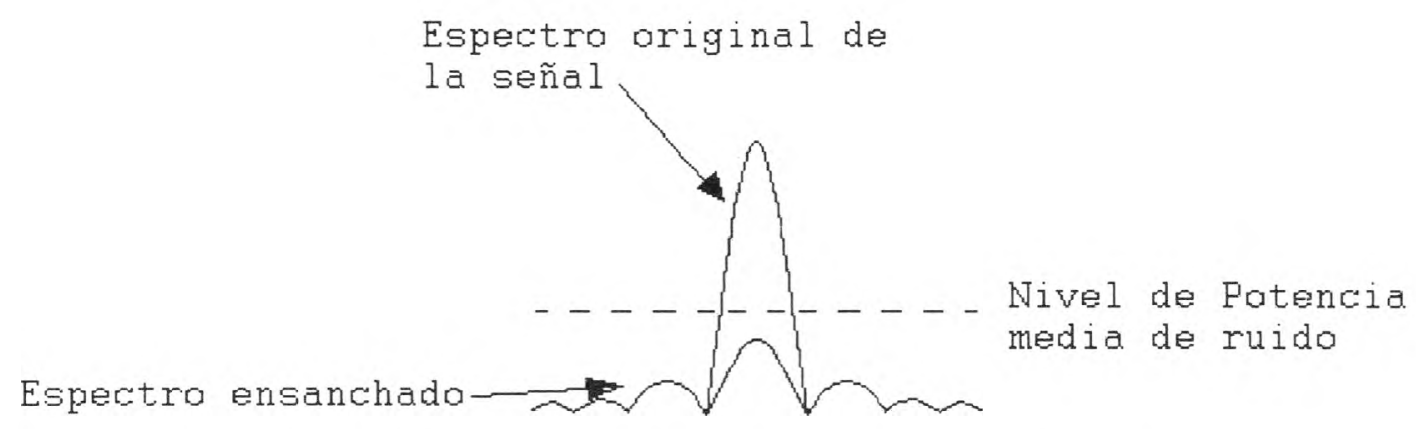

Figura 3. Espectro ensanchado por secuencia directa para protección de la componente analógica 
Tabla 1. Esquema de modulación

\begin{tabular}{|c|c|c|}
\hline Subsistema & Técnica propuesta & Características \\
\hline Modulación & $\begin{array}{l}\text { Híbrido Analógico-Digital de tasa } \\
\text { múltiple, } 4-16-64 \text { PSK con } \\
\text { componente radial analógica }\end{array}$ & $\begin{array}{l}\text { Ambas componentes representan tres } \\
\text { distintos niveles, dependiendo de su } \\
\text { importancia en la recuperación de la } \\
\text { señal. Permite reusar el ancho de banda } \\
\text { asignado con dos tipos de señales } \\
\text { distintos. Se obtiene una exelente } \\
\text { eficiencia en la transferencia de datos, } \\
\text { pues permite la obtención de } 2,4 \text { y } 6 \text { bits } \\
\text { de información por portadora modulada. } \\
\text { Con lo anterior, el receptor sería capaz de } \\
\text { adaptarse a las características de calidad } \\
\text { de la señal que recibe y, además, se } \\
\text { podrían construir receptores que se } \\
\text { adecuen específicamente a una de las } \\
\text { calidades, con lo que se disminuiría su } \\
\text { costo. }\end{array}$ \\
\hline
\end{tabular}

Tabla 2. Codificación de la señal

\begin{tabular}{|c|c|c|}
\hline Subsistema & Técnica propuesta & Características \\
\hline \multirow[t]{3}{*}{ Codificación de la señal } & $\begin{array}{l}\text { Componente Digital. Se protege a } \\
\text { través de un esquema de } \\
\text { modulación codificada TTCM } \\
\text { (Turbo Trellis Code Modulation). } \\
\text { Estos códigos tienen un excelente }\end{array}$ & $\begin{array}{l}\text { Las ganancias que presentan } \\
\text { ambas (de } 8.27 \text { y } 16 \mathrm{~dB} \\
\text { respectivamente) permiten la } \\
\text { transmisión a menores potencias. }\end{array}$ \\
\hline & $\begin{array}{l}\text { rendimiento cuando la relación } \\
\text { señal a ruido es relativamente baja } \\
\text { y se acerca al límite teórico de la } \\
\text { capacidad del canal propuesto por } \\
\text { Shannon. }\end{array}$ & $\begin{array}{l}\text { El uso de TTCM permite enviar } \\
\text { redundancia sin incrementar el } \\
\text { ancho de banda utilizado y sin } \\
\text { reducir la tasa de transmisión de } \\
\text { símbolos, mientras que las } \\
\text { secuencias PN del esquema DSSS }\end{array}$ \\
\hline & $\begin{array}{l}\text { Componente Analógica. Se obtiene a } \\
\text { partir del uso de una técnica DSSS } \\
\text { (Direct Sequence Spread- } \\
\text { Spectrum) a partir de códigos } \\
\text { seudo-aleatorios Kasami. }\end{array}$ & $\begin{array}{l}\text { permiten el envío de una gran } \\
\text { cantidad de información a } \\
\text { potencias bajas. Además, DSSS } \\
\text { permite el reuso de frecuencia } \\
\text { dentro de un área de cobertura. } \\
\text { Asimismo, pueden crearse redes } \\
\text { CDMA para enviar distintas } \\
\text { señales en el mismo ancho de } \\
\text { banda. }\end{array}$ \\
\hline
\end{tabular}

Para el sistema de radiodifusión se ha elegido el uso de turbo códigos con modulación de malla (Figura 4), que es una versión mejorada de los códigos de malla (Figura 5). El beneficio que conlleva su uso nos permite recuperar señales hasta de nivel 3 en donde otros esquemas sólo serían capaces de decodificar señales de nivel 1. Además su rendimiento no disminuye al utilizarse en receptores fijos, móviles o con gran cantidad de ecos. 


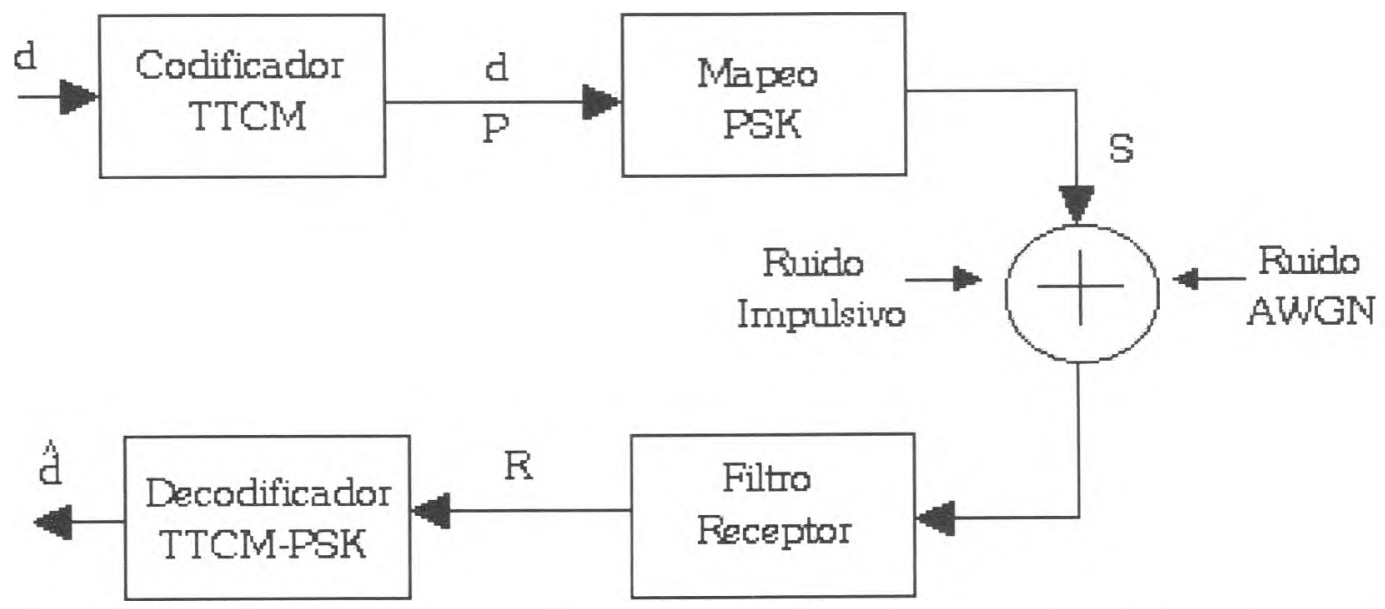

Figura 4. Configuración de los sistemas de codificación y decodificación TTCM

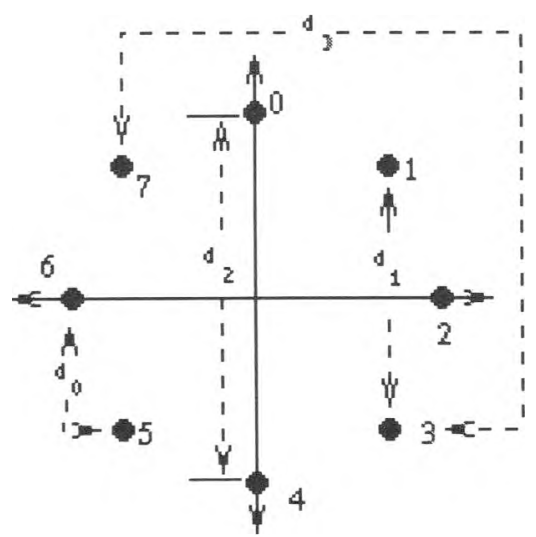

Figura 5. Transiciones válidas para modulación codificada de malla 8-PSK

\section{Ecualización}

A través del proceso de ecualización es posible filtrar la interferencia entre símbolos en el receptor. Sin embargo, mediante el uso de un ecualizador de error cuadrático medio basado en tomas de decisiones por realimentación, es posible también filtrar algo del ruido externo que llega al receptor, de tal forma que no sólo se pueda eliminar la interferencia, sino que puede mejorarse la relación señal a ruido y obtener así una mejor calidad de imagen y sonido (Figura 6).

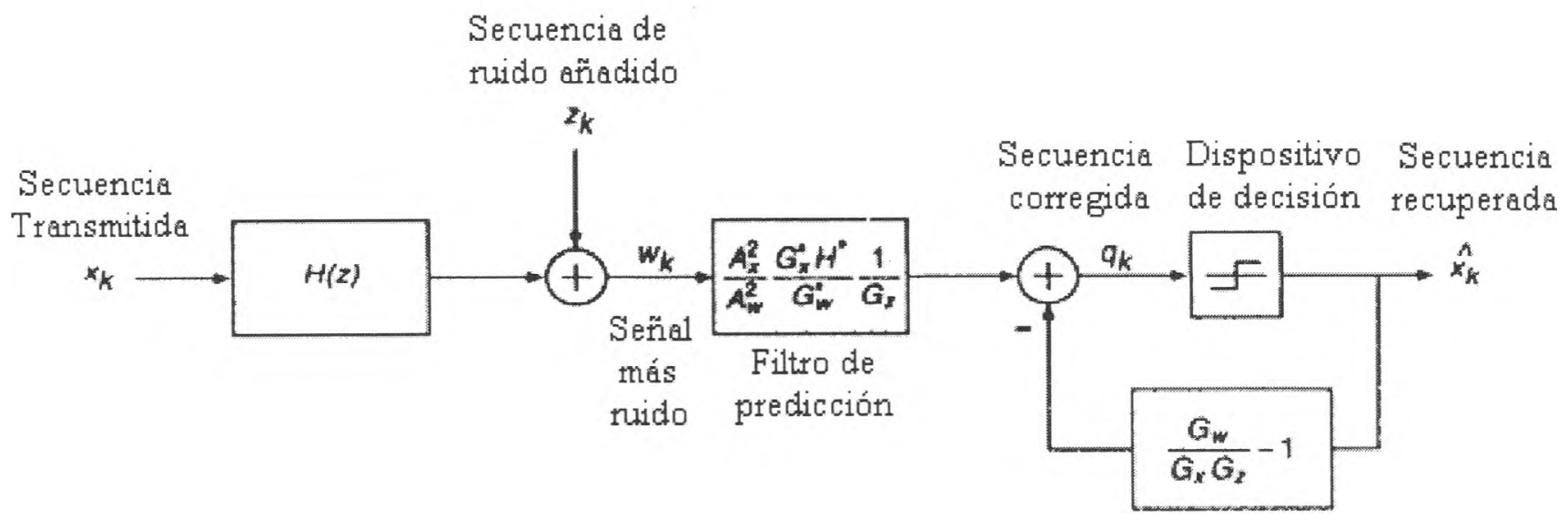

Figura 6. Ecualizador DFE-MSE para el canal de tiempo discreto $H(z)$ 
Tabla 3. Ecualización y caracterización del canal de transmisión

\begin{tabular}{lll}
\hline \multicolumn{1}{c}{ Subsistema } & \multicolumn{1}{c}{ Técnica propuesta } & \multicolumn{1}{c}{ Características } \\
\hline $\begin{array}{l}\text { Caracterización del canal de } \\
\text { transmisión y ecualización. }\end{array}$ & Se obtiene a partir del esquema de & Permite que el sistema elimine la \\
& ecualización DFE-MSE. & componente de interferencia entre \\
& símbolos provocada por las \\
& características del canal y \\
& beneficia en la reducción del ruido \\
& agregado a la señal sin disminuir \\
& la información transmitida por el \\
& sistema. \\
\hline
\end{tabular}

A través del uso de ecualización se puede encontrar la caracterización del canal, lo que en los sistemas convencionales se logra a través de señales de prueba. Sin embargo, esta última solución no es muy recomendable, pues reduce la tasa de transmisión de símbolos. Si se intentara contrarrestar esto utilizando menos señales piloto, se perdería confiabilidad en la caracterización obtenida. La tabla 3 resume las características principales del esquema de ecualización propuesto.

\section{Multicanalización y distribución de la señal}

La multicanalización de la señal se lleva a cabo en cada una de las componentes de la modulación. La componente digital lo hace por medio de un esquema de COFDM modificado, el cual permite obtener mejores resultados que en otros sistemas, mientras que la componente analógica se multicanaliza por medio de la técnica de CDMA utilizando el esquema DSSS con códigos Kasami.

Tabla 4. Multicanalización y distribución de la señal

Subsistema

Multicanalización.
Técnica propuesta

Componente Digital. A través de un esquema COFDM modificado.

Componente Analógica. A través de una técnica DSSS con códigos Kasami.

Distribución de la señal Celular a través de un esquema de red de frecuencia única.
Características

Con las modificaciones propuestas para COFDM, es posible obtener un rendimiento mayor al de los sistemas ATSC y DVB-T. Entre éstas se incluyen el uso de intercalación temporal y en frecuencia, así como el uso de TTCM en la componente digital.

El uso de códigos Kasami nos permite la obtención de una gran cantidad de secuencias $\mathrm{PN}$, lo que incrementa la capacidad del canal enormemente y permite escoger de entre las secuencias las que presenten un mejor comportamiento.

Con este esquema es posible conformar áreas de cobertura de tal forma que se adecuen óptimamente a las necesidades de la radiodifusora. Además, la transmisión a bajas potencias evita la interferencia de canal común y adyacente con otras radiodifusoras con áreas de cobertura cercanas. 
La distribución de la señal se realiza mediante células que transmiten la misma señal de forma simultánea, mediante el uso de SFN, lo que nos permite tener servicios de valor agregado. La componente digital será inmune a los ruidos mediante el uso de un intervalo de guardia, mientras que la componente analógica, conformada por DSSS, será interpretada correctamente por el receptor al utilizar únicamente la señal de mayor potencia ( sincronizándose con ella) y rechazar los ecos y retrasos en la señal. La tabla 4 resume las características de multicanalización y distribución dela señal.

\section{Sincronía y servicios de valor agregado}

Estos servicios se brindan por secuencias PN a través de CDMA. La sincronía puede ser de dos formas: por medio de una secuencia seudo-aleatoria y a través de un cálculo similar al utilizado por los GPS para sincronizarse con los transmisores. Como primera opción está la sincronización por medio de la asignación de una secuencia PN única a cada transmisor, en ella se transmite la posición de éstey es posible calcular la posición del receptor y su tiempo exacto. Sin embargo, es necesaria la presencia de al menos 4 receptores para que se logre obtener esta sincronía. Si las condiciones no lo permiten, se puede utilizar alguna de las secuencias PN enviadas para brindar la sincronía. La tabla 5 resume lo anterior.

\section{Transmisor}

En la figura 7 se muestra la estructura de un transmisor para el sistema de radiodifusión digital propuesto. Este esquema nos permite observar la estructura dentro de un sistema que consta de tres células. Cada una de estas células transmite la misma señal digital y casi la misma señal analógica, excepto por una secuencia PN que identifica a cada transmisor. Como se observa en dicha figura, se realizaron dos procesos paralelos para cada una de las componentes (analógica y digital), para su codificación de canal.

Tabla 5. Obtención de la sincronía de la señal

Subsistema

Sincronia

Servicios de Valor Agregado
Técnica propuesta

A través de la componente analógica de nivel 1 y del sistema de posicionamiento.

Sistema de Posicionamiento y Señal de reloj con exactitud cercana a la de un reloj atómico.

\section{Características}

Se utiliza el nivel perceptivo más alto de la multicanalización de la componente analógica a través de secuencias PN.

Para la recuperación del reloj por medio del sistema de posicionamiento, se requiere captar la señal de al menos 4 transmisores.

Con la obtención de señales de 3 transmisores, es posible obtener la posición exacta de un receptor móvil. En caso de que se contase con la señal de un transmisor adicional, sería posible recuperar una señal de reloj con exactitud similar a la de un reloj atómico. 
En el proceso de la componente analógica se envía una parte de los datos pertenecientes al nivel 1 a través de la red de distribución a cada una de las estaciones transmisoras. Estos datos son asignados a distintas secuencias a través de un acceso múltiple por división de código. Y se les añade la sincronía, además de una secuencia única que identifica a cada una de las estaciones transmisoras de la red. Mientras tanto, para la componente digital se utiliza un esquema similar a COFDM que consta del uso de un turbo código basado en modulación codificada de malla y la inserción de un código Reed-Solomon, luego la señal es distribuida a través de una red de datos a todas las estaciones transmisoras, "donde se relacionan ("mapean") los dos símbolos PSK que se transmitirán por cada portadora perteneciente a la multicanalización OFDM. Por último, en la etapa de amplificación y cambio de frecuencia se suman ambas componentes para enviarse a través del subsistema de antenas.

\section{Receptor}

El receptor se muestra, de forma gráfica, en la figura 8. La señal captada por la antena del aparato receptor deberá pasar por un filtro que limite la cantidad de ruido y de ancho de banda que entra a la etapa amplificadora. Dentro de ella se debe incluir un Amplificador de Bajo Ruido (LNA, Low-Noise Amplifier) que permita disminuir la producción de ruido de la etapa de cambio de frecuencia. Después de esto se deben separar ambas componentes para su posterior análisis. Esta separación se lleva a cabo al obtener la componente digital a partir del valor medio de cada una de las portadoras. La componente analógica será obtenida a

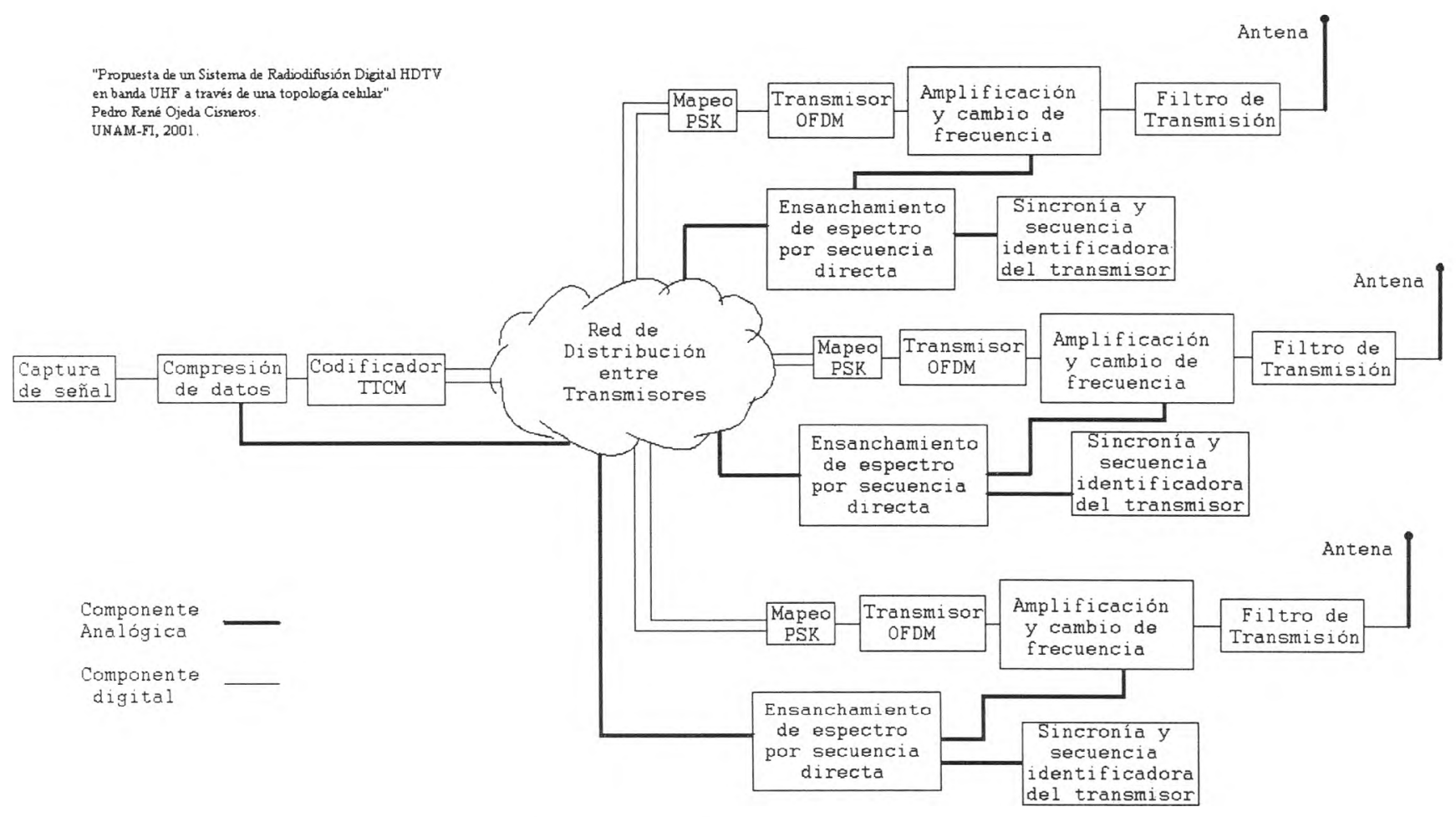

Fuigura 7. Esquema del sistema de transmisión 
través de un método de correlación con las secuencias seudo-aleatorias que portan información del nivel 1, la sincronía, y en caso de que se cumplan ciertas condiciones, los servicios de valor agregado. Además, a cada una de las secuencias seudo-aleatorias se les puede asignar un distinto valor perceptivo para mejorar la confiabilidad. La componente digital pasa por un proceso de ecualización para corregir los efectos producidos por el canal, como los ecos, y por medio del uso de un esquema DFE-MSE se puede disminuir en cierta forma también el ruido del canal, como lo demuestra el trabajo de Nicolas (1994).

En conjunto con el ecualizador se debe incluir el decodificador TTCM-PSK, al igual que en el caso de la combinación con modulación de malla (figura 6). Este sistema TTCM, basado en el trabajo de Koike y Ogiwara (1998), incluye dos decodificadores TCM, intercaladores de símbolos y también consta de un dispositivo de decisión basado en modulación PSK. Esta decodificación se debe hacer para cada una de las portadoras y la toma de la decisión se realiza con la concatenación de ellas, una vez que se han reordenado los símbolos enviados que fueron "aleatorizados" por medio de un proceso de intercalación. Esto le permite comportarse con gran eficiencia en distintos tipos de circunstancias como en aplicaciones de radiocomunicación fija, móvil y en presencia de mucho ruido, como ha sido demostrado en diversos trabajos, incluyendo el de Frengel (1999). En el proceso de descompresión se lleva a cabo la obtención de los distintos niveles de calidad de la señal, por medio de métodos que lo permitan. Los tres niveles propuestos en esta tesis brindan resoluciones distintas dependiendo del receptor y de las condiciones del medio en el que se encuentre.

NOTA: Las etapas de ecualización DFE-MSE y codificación TTCM-PSK no se deben conectar en cascada, sino de la forma en la que se especifica en el tema "DFE con modulación codificada" del trabajo realizado por Ojeda (2001).

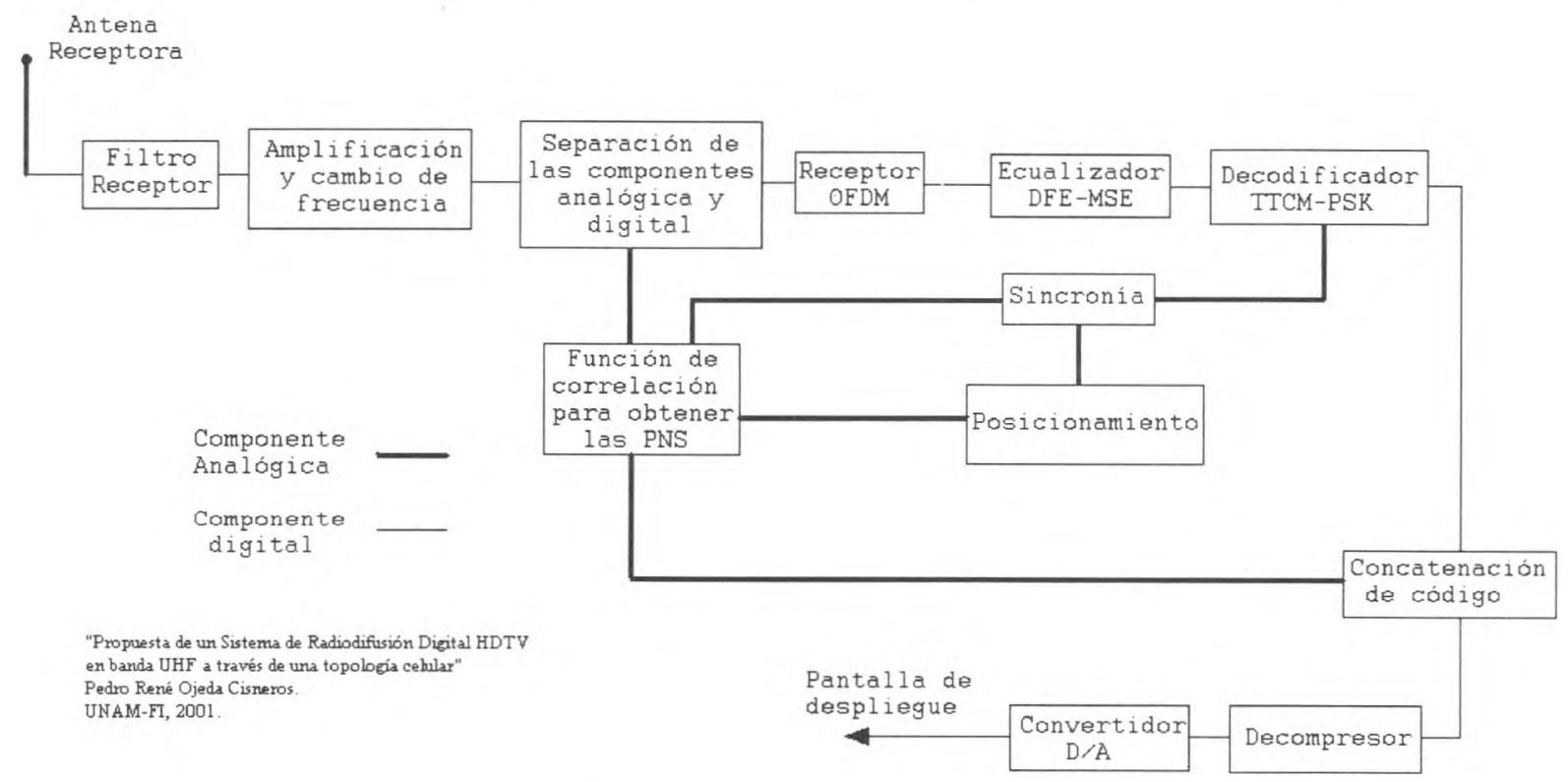

Figura 8. Esquema del aparato receptor 
Tabla 6. Comparación con otros sistemas

\begin{tabular}{|c|c|c|c|c|}
\hline Subsistema & Técnica propuesta & $\begin{array}{l}\text { Característica del } \\
\text { sistema }\end{array}$ & ATSC & DVB- $\mathrm{T}(\mathrm{BW}=6 \mathrm{MHz})$ \\
\hline $\begin{array}{l}\text { Tasa máxima de } \\
\text { Transmisión }\end{array}$ & $\begin{array}{l}\text { De tres niveles, } \\
\text { dependiendo de la } \\
\text { calidad de señal } \\
\text { obtenida por el } \\
\text { receptor y de las } \\
\text { características de } \\
\text { éste. }\end{array}$ & $\begin{array}{l}\text { Con la obtención de } \\
\text { los tres niveles de } \\
\text { señal, es posible } \\
\text { alcanzar velocidades } \\
\text { de hasta } 105.744 \\
\text { [Mbps]. }\end{array}$ & $\begin{array}{l}\text { Puede transmitir } \\
\text { tasas cercanas a los } \\
19.39 \text { [Mbps] (Eory, } \\
\text { 1996). Por sus } \\
\text { características, será } \\
\text { difícil aumentarla } \\
\text { sin la necesidad de } \\
\text { realizar cambios } \\
\text { radicales en el } \\
\text { estándar. }\end{array}$ & $\begin{array}{l}\text { Puede transmitir } \\
\text { tasas cercanas a los } \\
19.3 \text { [Mbps] } \\
\text { (Jacklin y } \\
\text { MacAvock, 1998). } \\
\text { Tiene la capacidad } \\
\text { de crecer a través de } \\
\text { variaciones en el } \\
\text { estándar. }\end{array}$ \\
\hline $\begin{array}{l}\text { Calidad máxima del } \\
\text { sistema. }\end{array}$ & $\begin{array}{l}\text { De tres niveles, } \\
\text { dependiendo de la } \\
\text { calidad de señal } \\
\text { obtenida por el } \\
\text { receptor y de las } \\
\text { características de } \\
\text { éste. }\end{array}$ & $\begin{array}{l}\text { 1600x900 píxeles, } \\
\text { con una } \\
\text { profundidad de } \\
\text { color de } 24 \text { bits } \\
\text { (True Color), } 60 \\
\text { cuadros por segundo } \\
\text { a resolución } \\
\text { completa y hasta } 8 \\
\text { canales de audio } \\
\text { con calidad superior } \\
\text { al disco compacto. }\end{array}$ & $\begin{array}{l}\text { Hasta } 1080 \text { líneas a } \\
30 \text { cuadros por } \\
\text { segundo y en } \\
\text { formato interlaced. }\end{array}$ & $\begin{array}{l}\text { Hasta } 1080 \text { líneas a } \\
30 \text { cuadros por } \\
\text { segundo y en } \\
\text { formato interlaced o } \\
\text { hasta } 720 \text { líneas a } \\
60 \text { cuadros por } \\
\text { segundo } \\
\text { (progressive). }\end{array}$ \\
\hline
\end{tabular}

\section{Máxima tasa de transmisión de los distintos niveles}

La componente digital de la modulación nos permite transmitir 2, 4 y 6 bits al mismo tiempo. Mientras tanto, la componente analógica puede transmitir varias secuencias y dentro de cada una de ellas puede transmitirse una cierta cantidad de información. A continuación, se realizará el diseño del sistema que pueda transmitir resoluciones de hasta $1600 \times 900$ píxeles, con una profundidad de color de 24 bits (True Color) y a 60 cuadros por segundo (completos) de forma progresiva, con lo que se asegurará una imagen de altísima calidad. Además, también se incluirá sonido de 8 canales (permite el uso del estándar Surround 7.1), cada uno de ellos con calidad superior a la del disco compacto. Para este diseño se supondrá que la señal recibida es de la suficiente calidad para poder interpretar datos de los tres niveles.

Para las condiciones anteriores tenemos que cumplir con una cierta cantidad de tasa de transmisión de bits que para la componente de video puede calcularse con la ecuación (1).

$$
\begin{aligned}
& T_{\text {transm }}=\left(R_{\text {horizontal }} \cdot R_{\text {vertical }}\right)[\text { pixeles }] \cdot \text { color } \\
& {[\text { bits } / \text { pixel }] \cdot \text { framerate }[\text { cuadros } / \text { segundo }]}
\end{aligned}
$$

Con lo anterior y sustituyendo valores tendremos que

$$
\begin{aligned}
& T_{\text {transm }}=(1600 \cdot 900)[\text { pixeles }] \cdot 24 \\
& {[\text { bits } / \text { pixel }] \cdot 60[\text { cuadros } / \text { segundo }]}
\end{aligned}
$$




$$
T_{\text {transm }}=2,073^{\prime} 600,000[\text { bits } / \text { segundo }]
$$

Para la componente de audio, la tasa de transmisión de bits requerida está definida por la ecuación (2).

$$
\begin{gathered}
T_{\text {transm }}=(\text { calidad })[\text { bits } / \text { muestra }] \cdot(\text { muestreo }) \\
{[\text { muestras } / \text { segundo }] \cdot(\text { canales de audio })}
\end{gathered}
$$

A partir de la ecuación anterior y sustituyendo los valores antes mencionados, tenemos:

$$
\begin{gathered}
T_{\text {transm }}=(16)[\text { bits } / \text { muestra }] \cdot(48000) \\
{[\text { muestras } / \text { segundo }] \cdot(8)} \\
T_{\text {transm }}=6^{\prime} 144,000[\text { bits } / \text { segundo }]=6.144[\mathrm{Mbps}]
\end{gathered}
$$

Con la utilización de MPEG-2 y AC-3, podemos tener tasas de compresión de hasta 75 y 16 veces, en video y audio, respectivamente, como lo refieren en sus trabajos Apostolopous (1991) y el Instituto Fraunhofer para Circuitos Integrados (2001). El uso del estándar MPEG-4 logra obtener tasas de compresión hasta 12 veces menores que para el caso de MPEG-2 (Wipro Technologies, 2001).

Si se utiliza el mínimo de ancho de banda necesario para transmitir el audio, únicamente en la componente digital del sistema se requerirían de al menos

$$
B W_{\text {audio }}=
$$

$\frac{(\text { tasa de transmisión })[\text { bits } / \text { segundo }]}{\text { Compresión } \cdot(\text { Tasa de transmisión por portadora })[\text { bits } / \mathrm{Hz}]}$

$$
B W_{\text {audio }}=\frac{6^{\prime} 144,000[\text { bits } / \text { segundo }]}{16.6[\mathrm{bits} / \mathrm{Hz}]}=64[\mathrm{kHz}]
$$

De tal forma que para video tenemos disponibles $B W_{\text {video }}=6[\mathrm{MHz}]-64[\mathrm{kHz}]$ correspondientes a la com-ponente digital, lo que nos permite una tasa de transmisión de

$$
\begin{gathered}
T_{\text {video }}=5.936[\mathrm{MHz}] \cdot 6[\mathrm{bits} / \text { portadora }] \cdot 1 \\
{[\text { portadora } / \mathrm{Hz}]=35.6[\mathrm{Mbps}]}
\end{gathered}
$$

Si se utilizan Códigos Kasami, se podrán obtener $2^{\mathrm{n} / 2} \cdot\left(2^{\mathrm{n}}+1\right)$ secuencias distintas $(v$. Tema 4.12.4). Si para el diseño, utilizamos un sistema de corrimiento de registros de 10 bits, tendremos $S=2^{10 / 2} \cdot\left(2^{10}+1\right)=32800$ secuencias distintas, de las cuales podremos escoger las necesarias para satisfacer nuestros requerimientos. Si consideramos transmitir datos a $64 \mathrm{kbps}$ con código Manchester, en cada secuencia PN, podríamos obtener una ganancia de codificación de

$$
\begin{gathered}
G=6[\mathrm{MHz}] /(2 \cdot 64[\mathrm{kHz}])=46.88 \Rightarrow \\
G_{d B}=16.7[\mathrm{~dB}]
\end{gathered}
$$

Para este diseño es necesario utilizar algunas de estas secuencias para transportar otros servicios, se pueden utilizar únicamente 10 de ellas para sincronía y otras 6 para posicionamiento. Aunque sólo se requeriría 1 de ellas para cada servicio, es 
posible utilizar más para servicios trascendentales, con el fin de obtener una mayor protección y brindar redundancia en el sistema, como se explica en el capítulo 4 de Ojeda (2001).

Si escogemos únicamente las 1020 secuencias que cumplan de mejor forma con las características de ortogonalidad, tendremos todavía $S_{\text {video }}=1015-10-10=1000$ secuencias, únicamente para transporte de video. Esto permitirá obtener de la componente analógica, un total de

$T_{\text {video }}=1000[$ secuencias $] \cdot 64[\mathrm{kbps} / \mathrm{secuencia}]=$

$$
64 \mathrm{Mbps}
$$

Con lo que sobran todavía 31785 secuencias sin utilizar. Sin embargo éstas pueden brindar redundancia en el sistema, así que se podrían asignar fácilmente 8 secuencias a cada señal de 64 kbps y aún así, quedar suficiente espacio para crecimiento futuro. De esta forma se tienen tres distintos niveles perceptivos de señal, dependiendo de la trascendencia de cada una de ellas dentro del sistema.

Si se suman las tasas de transmisión de las componentes analógica y digital (ecuaciones (4) y (6), se tiene un total de

$$
\begin{gathered}
T_{\text {video }}=T_{\text {analogica }}+T_{\text {digital }}= \\
64[\mathrm{Mbps}]+35.6[\mathrm{Mbps}]=99.6[\mathrm{Mbps}]
\end{gathered}
$$

De tal forma, que a partir de las ecuaciones (2) y (8), se tiene que la tasa de compresión requerida para el sistema es

$$
\text { Compresión }=\frac{2,073.6[\mathrm{Mbps}]}{99.6[\mathrm{Mbps}]}=20.81
$$

La cual es satisfecha cabalmente por el estándar MPEG-2 y aún más por MPEG-4, por lo que se demuestra que por medio de las técnicas elegidas para el sistema, es posible obtener la calidad descrita al principio de este tema.

Para el caso de que el receptor sólo fuera capaz de recuperar su señal con nivel 2, se alcanzaría fácilmente una resolución de 1280x720 píxeles a 60 cuadros por segundo y con una profundidad de color de 24 bits. Mientras tanto, el audio se reduciría a 5 canales con calidad superior a la de un disco compacto y un canal extra con calidad similar a la del FM estéreo.

Si el receptor únicamente recupera señal del nivel 1 , será capaz de obtener una imagen con resolución de $1080 \times 607$ píxeles a 60 cuadros por segundo (progressive) y con una profundidad de color de 24 bits. Los canales de audio se verían reducidos a sólo dos canales con calidad superior a la del disco compacto y un canal con calidad idéntica a la del CD. Si esto se combinara con un esquema de codificación Pro-Logic se podría obtener sonido envolvente (Surround) de tres canales.

Debido a la flexibilidad que nos permite este sistema, las características pueden ser modificadas dependiendo de lo que se considere más importante. Un ejemplo de lo anterior, es que se podrían ocupar secuencias PN para el transporte de audio y de otros niveles de señal de video, incrementando la tasa de transmisión, la calidad de la imagen y sonido a calidades aún mayores. Además se podrían utilizar la totalidad de estas secuencias seudo-aleatorias para la transmisión de datos de valor agregado, como información de programación o estado del clima. 


\section{Conclusiones}

Cada una de las técnicas aquí propuestas seha implementado de forma aislada para hacer pequeñas mejoras a diversos sistemas de comunicación, todas ellas han tenido gran éxito en el ámbito en el que operan. Con ellas, el sistema diseñado logra transmitir información a tasas de transmisión tan altas que permiten radiodifundir señales de calidad inigualable por los sistemas de transmisión actuales (analógicosy digitales).

Las pantallas de alta calidad deberán ser capaces de conmutar entre las distintas resoluciones existentes para desplegar la resolución seleccionada por el receptor, dependiendo de la señal que obtenga. Para el caso de receptores móviles, que generalmente obtienen una señal más degradada que para el caso de los receptores fijos, obtendrán la señal de nivel l (1080×607 píxeles a 60 cuadros por segundo) de la que podrían a su vez, obtener señales de menor resolución (espacial y temporal), ya que generalmente, son pantallas de menor tamaño.

Para la radiodifusora, este sistema también presenta una gran flexibilidad, económicamente hablando, pues le permite su crecimiento conforme vayan aumentando las necesidades y también dependiendo de la calidad de la señal que desee entregar dentro del área de cobertura. Con ello, se podría brindar inicialmente sólo la señal de nivel $1 \mathrm{y}$, conforme se requiera, aumentar la calidad de la señal incrementando un poco la potencia del transmisor. También se pueden tener áreas de cobertura de forma óptima que se adecuen a las características que se presenten dentro de ellas.

El uso de la combinación de Turbo códigos con Modulación codificada de malla y
Códigos Reed-solomon en la componente digital, permite incrementar la confiabilidad del sistema a niveles inigualables por ningún otro sistema existente en la actualidad, además de que nos brinda una alta ganancia de codificación (de hasta 8.27 $\mathrm{dB}$, a sólo $0.385 \mathrm{~dB}$ del límite teórico de Shannon, en presencia de AWGN), lo que se transforma en la necesidad de transmitir a potencias casi 7 veces menores de lo que normalmente se necesitaría. Esto, a la larga, permitirá a las empresas recuperar sus inversiones. También debe tomarse en cuenta la ganancia brindada por las secuencias PN en la componente analógica, que es de casi 47 veces. Ambas técnicas hacen un uso eficiente del espectro, la primera de ellas envía redundancia sin la necesidad de in- crementar el ancho de banda y sin reducir la tasa de símbolos enviados, mientras que la segunda permite el envío de una gran cantidad de secuencias PN que transportan datos de baja velocidad con una alta ganancia.

Así también, a través de la topología celular los transmisores no necesitan transmitir a potencias altas, ya que su región de cobertura es mucho menor que en el caso de los esquemas de un solo transmisor y no se tiene que dar servicio a zonas en las que no se hallen receptores que puedan decodificar la señal enviada por la radiodifusora, por lo que no se tiene que invertir en infraestructura en dichos lugares.

La transmisión a bajas potencias también nos permite que las distorsiones provocadas por los amplificadores de transmisión sean bajas, pues se encuentran operando en regiones lineales, por lo que no existe ruido por intermodulación.

Por todo lo anterior, este sistema contrarresta todos y cada uno de los 
problemas inherentes a la banda UHF, brindando la más alta calidad de audio y video que ningún otro sistema de radiodifusión de televisión terrestre haya propuesto.

Para concluir, en la tabla 6 se muestra una comparación entre el sistema demostrado y los sistemas actuales.

\section{Referencias}

Koike-Kiyoyuki y Haro-Ogiwara (1998). Application of Turbo TCM Codes for Impulsive Noise Channel. IEICE Trans. Fundamentals, Vol. E81-A No. 10.

Apostolopoulos J.G. (1991). Coding of the Motion-Compensated Residual for an All-Digital HDTV System. Tesis de Doctorado en Ingeniería Eléctrica, Massachusetts Institute of Technology, Massachussets, Estados Unidos.

Frenger Pål (1999). Turbo Decoding on Rayleigh Fading Channels with Noisy Channel Estimates.

Nicolas-Julien J. (1994). Investigation of Coding and Equalization for the Digital HDTV Terrestrial Broadcast Channel. Tesis de Doctorado en Ingeniería Eléctrica, Massachusetts
Institute of Technology, Massachussets, Estados Unidos.

Ojeda P.R. (2001). Propuesta de un sistema de radiodifusión digital HDTV en banda UHF a través de una topología celular. Tesis de Licenciatura en Ingeniería, Universidad Nacional Autónoma de México, Ciudad Universitaria, México.

Eory-Frank (1996). Performance Assessment of the ATSC Transmission System, Equipment and Future Directions.

http://www.digitalspotnews.com/cgibin/news/en/topics/details/atsc/ATSCVSB-AHG-IR-Draft l.html

Fraunhofer Institute for Integrated Circuits IIS-A (2001). MPEG Audio Layer-3.

http://www.iis.fraun-ofer.de/amm/tec hinf/aac/index.html

Jacklin M. y MacAvock P. (1998). DVB Comments on the DVB-T Digital Terrestrial Broadcasting Standar. http://www.wmr.com/html/Facts_abo ut_DVB-T.htm

Wipro Technologies (2001). MPEG-4 Video Decoder.

http://www.itpapers.com/cgi/PSumm aryIT.pl? paperid $=205688$ scid $=155$ 


\section{Semblanza de los autores}

Pedro René Ojeda-Cisneros. Obtuvo su título con mención honorífica como ingeniero en telecomunicaciones, con la tesis Módulo de dispositivos de microondas y ópticos, en la Facultad de Ingeniería, UNAM. Se hizo merecedor a la Medalla al Mérito Universitario "Gabino Barreda" y el reconocimiento de la ANFEI a los Mejores Egresados de Ingeniería del País, como el mejor egresado de la Facultad de Ingeniería, UNAM del año 2000; asimismo perteneció al Programa de Alto Rendimiento Académico. Actualmente trabaja en la Red Corporativa de Datos Telmex.

Salvador Landeros-Ayala. Egresó de la Facultad de Ingeniería, UNAM, con el título de ingeniero mecánico electricista en el área de comunicaciones. Cursó la maestría en ciencias de la ingeniería en telecomunicaciones en la Universidad de Pennsylvania, Estados Unidos; posteriormente, obtuvo el grado de doctor en ingeniería eléctrica en la Facultad de Ingeniería de la UNAM. Ha escrito artículos que se han presentado en congresos internacionales en Estados Unidos, Francia, España, Centro y Sudamérica. Fue miembro del Comité de Becas del CONACYT, director del Sistema de Satélites Nacionales y jefe de la División de Ingeniería Eléctrica. Actualmente es jefe de la División de Estudios de Posgrado de la Facultad de Ingeniería de la UNAM.

Rodolfo Neri-Vela. Realizó los estudios de ingeniero mecánico electricista y se especializó en telecomunicaciones y electrónica en la Facultad de Ingeniería, UNAM. En 1976, obtiene su maestría en telecomunicaciones en la Universidad de Essex, Inglaterra, becado por el Consejo Británico. Tres años después, obtuvo el grado de doctor en radiación electromagnética aplicada por la Universidad de Birmingham, Inglaterra, como becario del Consejo Nacional de Ciencia y Tecnología (CONACYT). En 1985, se convirtió en el primer astronauta mexicano al participar en la misión 61-B de la NASA de los EU y orbitar la Tierra 109 veces. Actualmente imparte cátedra en el Departamento de Telecomunicaciones de la Facultad de Ingeniería. 